\title{
Tecnologia educacional no processo de formação de enfermeiros
}

\section{Educational technology in the nurse graduation process}

\author{
Luis Felipe Pissaia, ${ }^{1}$ Arlete Eli Kunz da Costa, ${ }^{1}$ Márcia Jussara Hepp Rehfeldt, ${ }^{1}$ Claudete Moreschi ${ }^{1}$ \\ 'Centro Universitário Univates, Lajeado, RS, Brasil.
}

Recebido em: 14/01/2017 / Aceito em: 24/05/2017 / Publicado em: 30/06/2017

Ipissaia@universo.univates.br

\section{RESUMO}

Objetivo: o presente estudo possuiu o objetivo de identificar as percepções de enfermeiros acerca do contato com tecnologias educacionais durante sua graduação. Método: a pesquisa é descritivo-exploratória, de caráter qualitativo, realizada por meio da aplicação de questionário, a seis enfermeiros atuantes em uma unidade de internação clínica geral de um hospital de médio porte, localizado no Vale do Taquari, região central do estado do Rio Grande do Sul/RS, Brasil. Resultados: constatou-se, com base nos relatos dos profissionais que, durante a realização de seus cursos foram utilizadas diversas tecnologias incorporadas às metodologias de ensino existentes, tanto por meio de equipamentos e softwares utilizados em laboratórios, quanto por técnicas de ensino diferenciadas empregadas pelos docentes em diferentes momentos. Verificou-se que, durante as disciplinas foi contextualizada a aplicação de tecnologias no campo prático, em estágios e possíveis locais de trabalho, fortalecendo o contato com distintas ferramentas enquanto acadêmico. Os enfermeiros consideraram importante o contato com diferentes tecnologias, durante a graduação, como forma de preparação acadêmica e profissional, possibilitando uma vivência única e abrangente sobre o espaço de atuação do enfermeiro, principalmente, enquanto gestor de unidade de saúde. Considerações finais: considera-se pertinente a utilização de tecnologias em sala de aula como meio de facilitar os processos de aprender e apreender frente à problematização e reflexão dos estudantes. Verifica-se a necessidade do uso de tecnologias nos cursos de graduação em enfermagem como forma de qualificação do futuro profissional, exercendo assim, uma assistência de qualidade e resolutiva à população.

Palavras-chave: Tecnologias em Saúde; Instituicões de Ensino; Enfermeiro.

\begin{abstract}
Objective: the present study aims to identify the perceptions that nurses have about having contact with educational technologies during their graduation. Method: this is a descriptive-exploratory research of qualitative characteristic carried out through a questionnaire applied to six nurses in active work at a general clinic unit from a medium sized hospital located in Vale do Taquari, central region of the state of Rio Grande do Sul (Brazil). Results: according to the professionals' statements it was found that several technologies were used throughout their courses, incorporated with the existing teaching methodologies, both through of equipment and software used in the laboratory and through differentiated teaching techniques employed by the faculty at different instances. It was found that during the discipline the application of technologies was contextualized in the practical field, in internships and possible work locations, leading to the strengthening of the contact with different tools as undergraduates. The nurses deemed the contact with different technologies during graduation is important as a way for academic and professional preparation that allows for a single and comprehensive experience on nurses' space of actuation, especially as managers of a health unit. Closing remarks: it is considered relevant the use of technologies in the classroom as a means of facilitating the processes of learning and reflection of the students. There is a need to use technologies in nursing undergraduate courses as a way of qualifying the professional future, thus providing quality and resolute assistance to the population.
\end{abstract}

Keywords: Health Technologies; Health Institutions; Nurse. 


\section{INTRODUÇÃO}

A inserção de tecnologias em sala de aula na área da saúde trouxe consigo inúmeras modificações na educação, principalmente com o uso das chamadas tecnologias educacionais que revolucionaram os processos de ensino e de aprendizagem.1,2 A introdução destes modelos educacionais contribuiu para uma nova forma de produzir conhecimento, por meio da manipulação de diferentes formas de ensino, fomentando a problematização e a formação profissional tecnológica em saúde. ${ }^{2}$

A utilização de um modelo de ensino tecnológico influencia diretamente nos campos de trabalho dos profissionais de saúde, o que pré-dispõe a necessidade de capacitação que vise suprir as demandas nos diferentes contextos assistenciais. ${ }^{3} \mathrm{Na}$ área da enfermagem, as utilizações de novas metodologias de ensino possibilitam a compreensão de diferentes cenários através de tecnologias que reproduzem vivências educacionais promovidas por meio de Ambientes Virtuais de Aprendizagem (AVA). ${ }^{1,4}$

No atual contexto global, a necessidade de comunicação tornou-se algo imprescindível para a disseminação e apreensão do conhecimento, tornando-se necessária a utilização de tecnologias no ensino como forma eficaz e incentivadora de novas práticas em sala de aula. ${ }^{5}$ A implantação de objetos tecnológicos nos cenários educacionais deve obedecer a contextualização proposta, permitindo a autoaprendizagem por meio de técnicas de ensino que promovam sua relevância, durante a formação acadêmica. ${ }^{2,5,6}$

Para os profissionais da área, as diferentes tecnologias disponíveis fomentam a tomada de decisão correta, por estarem diretamente ligadas aos processos de gestão dos serviços de saúde e desencadearem a qualificação da assistência por meio de um controle de suas práticas. ${ }^{6,7}$ Os objetos tecnológicos fazem parte do dia a dia profissional, desempenhando um papel importante enquanto auxiliares na prestação de serviços de saúde, inferindo na utilização de ferramentas que abrangem o cuidado em sua plenitude. ${ }^{8}$

A incorporação de modelos tecnológicos na formação do profissional direciona a uma construção atualizada dos modelos tradicionais de ensino, o que qualifica o perfil proativo, oferecendo um arcabouço diferenciado de recursos que possibilitam sua utilização nos diferentes campos acadêmicos e profissionais. ${ }^{9}$ Para tanto, o seu uso no ensino torna-se crescente, colaborando com as novas demandas educacionais de disponibilidade de recursos, o que reflete diretamente na utilização de diferentes tecnologias em ambiente social e comunitário. ${ }^{8,10}$

As tecnologias tornam-se, portanto, presentes na formação acadêmica dos profissionais, integrando modelos diferenciados de técnicas de ensino, e incentivando a utilização de recursos tecnológicos. ${ }^{11,12} \mathrm{~A}$ qualificação acadêmica interfere na disponibilidade de uma assistência efetiva e resolutiva nos serviços de saúde, contribuindo para uma prática livre de estigmas, com uma visão ampliada do ser humano e da comunidade..$^{12,13}$

Desta forma, o presente estudo possui o objetivo de compartilhar as percepções de enfermeiros acerca do contato com tecnologias educacionais, durante sua graduação.

\section{MÉTODO}

A pesquisa é descritivo-exploratória, de caráter qualitativo, realizada por meio da aplicação de questionário, a seis enfermeiros atuantes em uma unidade de internação clínica geral de um hospital de médio porte. O referido campo de estudo localiza-se no Vale do Taquari, região central do estado do Rio Grande do Sul/RS, Brasil, sendo que a unidade estudada presta assistência a clientes por meio de convênio com o Sistema Único de Saúde (SUS) e sua estrutura física possui 25 leitos de internação.

Os profissionais foram selecionados por meio de critérios de inclusão estabelecidos previamente pelo projeto de pesquisa, ficando restrita a participação somente de enfermeiros atuantes na referida unidade há um ano ou mais, em todos os turnos de trabalho. Após identificar os profissionais que contemplaram os critérios estabelecidos, os pesquisadores entraram em contato, individualmente, convidando-os a participar da pesquisa e, neste momento, foram explicados os objetivos e os critérios éticos, que envolvem o estudo.

Após o aceite em participar da pesquisa realizou-se um agendamento para a aplicação do questionário conforme disponibilidade dos profissionais. Para tanto, a coleta de dados ocorreu durante o mês de fevereiro de 2016. Os diálogos foram individuais, em uma sala isolada, visando à garantia da privacidade e sigilo das informações.

Utilizou-se como instrumento de coleta de dados um questionário aberto, com questões semiestruturadas desenvolvido pelo grupo de pesquisadores. As questões buscaram identificar o perfil pessoal, por meio da idade, sexo e tempo de trabalho na instituição. As demais questões foram: (1) Em seu curso houve a utilização de tecnologias educacionais incorporadas à metodologia de ensino? Quais?; (2) Os docentes contextualizavam a utilização de ferramentas tecnológicas em relação ao mercado de trabalho do enfermeiro? e (3) Quais as suas percepções sobre o contato com tecnologias, durante a graduação em relação a atuação profissional?

Antes de iniciar os diálogos foram lidos os Termos de Consentimento Livre e Esclarecido (TCLE) e após assinados pelo participante e pelo pesquisador em duas vias de igual teor. Os nomes dos profissionais foram preservados pelo codinome "Enf." seguido por números aleatórios de 1 a 6 sorteados pelos pesquisadores. Os momentos de diálogo tiveram uma duração média de 30 minutos. Seus áudios foram gravados e, posteriormente, transcritos e analisados, conforme propõe a Análise Temática de Conteúdo de Minayo. ${ }^{14}$

De forma especial, este trabalho foi desenvolvido por meio do projeto de pesquisa "Impacto das tecnologias da informática no processo de Sistematização da Assistência de Enfermagem em uma instituição hospitalar do Vale do Taquari/RS, Brasil". O estudo possui aprovação da instituição hospitalar para realização da pesquisa, recebendo autorização pela Pró-Reitoria de Ensino e aprovado pelo Comitê de Ética em Pesquisa (COEP) do Centro Universitário Univates, por meio da CAAE $n^{\circ}$ 49947715.3.0000.5310. Neste projeto, contemplou-se a Resolução 466/2012 do Conselho Nacional de Saúde, que direciona os aspectos éticos que envolvem pesquisas com seres humanos. 


\section{RESULTADOS E DISCUSSÃO}

Com base nos dados coletados sobre o questionário de identificação do perfil profissional, constatou-se que os seis entrevistados são do sexo feminino. Quanto a sua faixa etária, verificou-se uma variação entre 26 a 48 anos de idade. Já, quanto ao tempo de trabalho na instituição variou entre um ano e meio a 10 anos completos.

A enfermagem possui um enraizado histórico de participação das mulheres na prestação de assistência. Em seus primórdios, como meio de abnegação religiosa, no entanto, atualmente esta característica se mantém visível. A totalidade de profissionais do sexo feminino se assemelha a outro estudo realizado em ambiente hospitalar com dezesseis profissionais em 2015, onde foi realizado o mesmo achado. ${ }^{15} \mathrm{Em}$ outro estudo publicado em 2015, identificou-se que a faixa etária dos profissionais entrevistados era dos 20 aos 60 anos de idade, mantendo-se pareada com a encontrada neste estudo dos 26 aos 48 anos. ${ }^{16}$

A partir da primeira pergunta (1) Em seu curso houve a utilização de tecnologias educacionais incorporadas à metodologia de ensino? Quais? Os entrevistados relataram que tiveram contato com tecnologias durante seus cursos de graduação, de diferentes maneiras, sendo incorporadas às aulas majoritariamente, por meio de projeções de textos, imagens e vídeos com o auxílio de equipamentos disponíveis em sala de aula, bem como a utilização de softwares específicos utilizados durante aulas práticas em laboratório. Ainda sobre a inserção de tecnologias em seu ensino, os profissionais destacaram a utilização de diferentes técnicas de ensino, como estudos de caso, simpósios, debates e resolução de problemas, conforme as falas:

“Houve sim, muitos métodos que hoje eu posso dizer que foram tecnológicos; a professora usava os slides, sabe o PowerPoint e isso auxiliava muito para os alunos aprenderem, ainda hoje, por que sei que os professores do meu filho também usam dessa maneira" (Enf. 2).

“Os meus professores, não todos, mas a maioria levava as turmas pros laboratórios né, e era muito bom quando era só da enfermagem, que daí a gente mexia naqueles computadores e sistemas que eram usados, além dos bonecos que tinham para as práticas nos laboratórios e nas salas, quando o professor levava" (Enf. 3).

"Pelo que eu lembro quase todos usavam alguma coisa assim nova pra dar aula, eu gostava muito quando passavam vídeos, principalmente aqueles de técnicas que às vezes no boneco é muito diferente de quando na pessoa mesmo, além disso, faziam sempre os simpósios, assim que a professora chamava, porque a gente ficava em círculo e discutia algum assunto e todos falavam isso eu acho legal" (Enf. 5).

“No meu curso sempre tinha alguma coisa diferente nas aulas, sabe aquelas disciplinas que temos que fazer com outros cursos, acha que ainda hoje deve ser assim, a gente se juntava em grupos e o professor projetava um texto e a gente lia e discutia; isso dá pra aprender muito, ainda lembro os estudos de caso também que a gente resolvia nos laboratórios valendo nota" (Enf. 6).
A utilização de recursos tecnológicos em sala de aula vem tomando forma nos diferentes espaços de ensino, desde a escolarização primária até a superior, instrumentalizado pela disponibilidade dos alunos em inovar e aprender, conforme estudo colaborativo realizado em 2016 com alunos portadores de deficiência visual no estado do Mato Grosso do Sul e outros estudos semelhantes realizados no mesmo ano.,17 $\mathrm{A}$ aplicação de técnicas de ensino como tecnologias educacionais na área da saúde diferencia-se dos modelos tradicionais de aprendizagem, fomentando a construção de novas formas de ensinar e aprender, compondo uma maneira ampliada e eficaz de reflexão aos acadêmicos conforme vivenciado em um estudo a partir da produção de portfólios em turmas de medicina, publicado neste ano de 2017.18,19

Já, quanto à segunda pergunta realizada (2), os docentes contextualizavam a utilização de ferramentas tecnológicas em relação ao mercado de trabalho do enfermeiro? Os profissionais relataram que os docentes utilizavam várias ferramentas em diferentes momentos, recebendo destaque para as aulas práticas desenvolvidas a partir de protótipos de moldes anatômicos e com a presença dos demais equipamentos necessários para a realização de procedimentos. Ainda sobre o contato de tecnologias, os entrevistados evidenciaram a utilização de diversos Sistemas de Informação e Comunicação (SIS), que eram apresentados e contextualizados em sala de aula e após foram utilizados em campos de estágio, durante o tempo em que se desenvolviam as práticas, conforme os relatos:

"Faz anos que fiz a minha faculdade, mas ainda lembro que a professora explicava todos os sistemas do Sistema Único de Saúde (SUS), sabe? Aqueles que se usa pro cadastro e também pra lançar os exames, e quando a gente ia em grupos nos postos fazer estágio, a gente já sabia fazer as coisas e ficava fazendo direto no computador; era bem mais fácil. E ajudou sim, porque logo depois de me formar eu fui trabalhar no posto e sabia o que fazer" (Enf. 1).

"Certamente que ajuda, pois a gente sempre ficava algumas aulas nos laboratórios; a gente chamava de aulas práticas e lá a turma fazia os procedimentos; daí tinha os bonecos e o material e tu se virava. A professora dava o kit pra sondagem e deixava nós com a boneca e ficava olhando se fazia certo, e isso foi bom, que todos aprendiam" (Enf. 2).

"A professora de gestão sempre falava dos sistemas que iríamos usar pra trabalhar no hospital; ela mostrava nas lâminas tudo e ensinava como calcular o pessoal, fazer as escalas e controlar os materiais com as planilhas do computador, daí quando fui fazer o meu estágio curricular cheguei lá e era tudo igual, ainda é hoje na grande maioria dos lugares e foi mais fácil. Quando eu entrei aqui, chegou uma colega nova também em outro setor e ela veio de outra faculdade e não sabia fazer nada dos sistemas e eu já sabia por que tinha aprendido antes na sala e no estágio" (Enf. 4).

“Sim mostravam na sala de aula tudo, não em todas as disciplinas, mas faziam sim, davam exemplos e depois eu via nos estágios, principalmente com os programas que tem no posto; nunca mais vi 
então nem lembro o nome direito, mas a professora explicava na sala e depois a enfermeira que supervisionava o estágio explicava de novo no lugar. Ainda no primeiro dia de estágio a supervisora fazia no laboratório e o grupo usava os bonecos pra fazer todos os procedimentos [...]" (Enf. 5).

Para Almeida e Silva, ${ }^{20}$ a introdução de tecnologias em diversos cursos da área da saúde, seguem o ritmo da sociedade contemporânea de incorporação tecnológica e comunicativa, trazendo sob suas concepções uma aproximação entre docentes e discentes conferindo uma maior qualidade ao ensino. Os achados, neste estudo, também colaboram com os encontrados em uma pesquisa realizada entre 2011 e 2012 com alunos do quarto semestre de enfermagem de uma universidade da região nordeste, sendo que a introdução de novas técnicas de ensino, visando ao ensino de conteúdos específicos de enfermagem, possibilitam momentos de reflexão e problematização, assegurando, assim, a aprendizagem ao grupo escolar. ${ }^{21}$

Para a terceira pergunta constante no questionário, (3) Quais as suas percepções sobre o contato com tecnologias durante a graduação em relação à atuação profissional? Os entrevistados consideraram importante o contato com diferentes tecnologias educacionais durante a graduação, sendo um diferencial para sua formação, conferindo capacitação perante os demais. Outro aspecto relevante que os profissionais levantaram foi o auxílio que os recursos tecnológicos utilizados em sala de aula conferiram para o entendimento e realização dos processos de gestão de unidade de saúde, principalmente quanto à utilização de ferramentas computacionais.

"Esse contato com tecnologias é essencial ho-
je, e sabe que estava conversando com outros
colegas são poucas universidades que ensinam
isso, e que usam na sala de aula. Pra mim, isso
foi um diferencial, que se destacou depois que
me formei, pensa comigo, em um concurso que
eu fiz era a única que tinha experiência com o
programa que o hospital usa, mas isso por que
tinha aprendido na sala e nos estágios" (Enf. 1).

“Tudo o que o professor usou foi importante, no momento, às vezes eu olhava espantada e não entendia, mas hoje trabalhando há anos eu vejo que fez muita diferença, as tabelas, os projetos que eles ensinavam as imagens tudo projetado com o Datashow. E ainda entender a importância que os programas têm pra comunicação entre a equipe e tudo mais; sem dúvidas isso me fez planejar melhor, aprendi a gestão assim não precisei fazer pós" (Enf. 3).

“Sim fez diferença, agora pensando no hoje, alguns professores sabiam usar mais que outros, mas ajudaram sim; na minha época ainda não se usava tanto o computador; então eles ensinavam a fazer planilhas com as escalas da equipe, pra controle de material, até da medicação que ficava no setor eu fazia isso, sem dúvidas isso melhorou" (Enf. 4).

"A minha formação foi toda diferenciada com isso, até lembrei agora que o professor de uma disciplina comentou que se tratava de metodologia ativa aquilo que ele fazia de diferente, todos gostavam e aprendiam muito, e tanto na sala, quanto aqui no hospital, quando ainda fazia estágio isso foi muito importante" (Enf. 6).

As atividades práticas de ensino, fora do ambiente da sala de aula, também foram caracterizadas como relevantes em uma pesquisa realizada com alunos de medicina da Universidade Federal do Amapá, durante o primeiro semestre de 2015, pontuando além da contextualização comunitária, a necessidade de ensino tecnológico, visando sua inserção profissional, o que reafirma os relatos encontrados neste estudo. ${ }^{22,23} \mathrm{Na}$ área, a indução a momentos de criatividade e imaginação de casos são fortalecidos pelo apoio tecnológico, quebrando barreiras e incentivando sua utilização, principalmente na área da gestão. Esta afirmação também foi reforçada por uma revisão de literatura realizada por pesquisadores do Instituto Politécnico de Viseu, Portugal, que mencionam pontos semelhantes aos destacados. ${ }^{23,24} \mathrm{Na}$ área da enfermagem, ainda observam-se dificuldades em inserir tecnologias em seu meio, tanto no ensino, quanto na prática profissional devido a preconceitos estabelecidos de cunho cultural que receiam a uma aversão ao seu propósito. Neste sentido, movimentos de libertação podem ser vistos como aqueles que incentivam a educação continuada por meio do uso de AVA. ${ }^{17,25,26}$

\section{CONSIDERACÕES FINAIS}

A partir da análise dos resultados, considera-se este estudo inovador para a área de formação, por meio do ensino tecnológico, devido à dificuldade em contextualizar os achados com estudos recentes em nível nacional e internacional, tornando-o diferenciado. Neste sentido, verificou-se que estão sendo realizadas modificações nesta área, adaptando-se a formação integral do futuro profissional, ao cenário tecnológico contemporâneo.

Compreendeu-se que o ensino tecnológico abarca uma gama infindável de metodologias e ferramentas passíveis de serem exploradas e introduzidas na área, especialmente na utilização de técnicas de ensino, que desafiem o aluno a vivenciar o futuro profissional. Considera-se pertinente a utilização de tecnologias em sala de aula como meio de facilitar os processos de aprender e apreender os conteúdos, bem como sua importância frente à problematização e reflexão dos indivíduos, ainda na posição de aluno.

A incorporação de tecnologias nos diferentes contextos acadêmicos investigados influenciou diretamente na formação profissional, conferindo uma distinção ao futuro profissional em suas áreas de atuação, fortalecendo principalmente os processos de gestão de serviços, aliados a recursos tecnológicos. Para tanto, verifica-se a necessidade do apoio de tecnologias durante a trajetória acadêmica, como forma de preparação para a atuação, bem como a qualificação de suas práticas conferindo uma assistência de qualidade e resolutividade à população.

Este estudo alavancou a necessidade de realização de novas pesquisas sobre a aplicação de tecnologias em sala de aula, não somente na graduação em enferma- 
gem, mas nos demais cursos que compreendem a área da saúde. Com a realização destes estudos, espera-se compreender não somente a utilização de tecnologias em cursos de graduação isolados, mas seu real impacto no trabalho multidisciplinar em saúde, fato este, que se tornou um limitante da presente pesquisa.

\section{REFERÊNCIAS}

1. Cyrino EG, Pinto HA, Oliveira FPD, Figueiredo AMD, Domingues SM, Parreira CMDSF. Há pesquisa sobre ensino na saúde no Brasil?. ABCS health sci 2016;40(3). doi: 10.7322/abcshs.v40i3.787

2. Booth A, Noyes J, Flemming K, Gerhardus A, Wahlster P, Van Der Wilt GJ, Rehfuess E. Guidance on choosing qualitative evidence synthesis methods for use in health technology assessments of complex interventions. 2016.

3. Goes TM, Polaro SHI, Gonçalves LHT. Cultivo do bem viver das pessoas idosas e tecnologia cuidativo-educacional de Enfermagem. Enfermagem em Foco 2016;7(2).

4. Cohen EL, Hoffner C. Finding meaning in a celebrity's death: The relationship between parasocial attachment, grief, and sharing educational health information related to Robin Williams on social network sites. Computers in Human Behavior 2016;65:643-650. doi: 10.1016/j. chb.2016.06.042

5. Rocha PF, Warmling CM, Toassi RFC. Preceptoria como modalidade de ensino na saúde: atuação e características do preceptor cirurgião-dentista da atenção primária. Saberes Plurais: Educação na Saúde 2016;1(1).

6. Wartella E, Rideout V, Montague H, Beaudoin-Ryan L, Lauricella A. Teens, health and technology: A national survey. Media and Communication 2016;4(3). doi: 10.17645/mac.v4i3.515

7. Gitahy RRC, Silva JP, Terçariol AAL. O uso das tecnologias de informação e comunicação aplicadas como tecnologia assistiva na construção do conhecimento dos alunos com deficiência visual que frequentam as salas de recursos multifuncionais. Revista Ibero-Americana de Estudos em Educação 2016;11(1). doi: 10.21723/riaee.2016.v11.n1.p111

8. Sant'Anna RMD, Escudeiro CL, Ferreira SCM, Teixeira MLDO, Castelo Branco EMDS. Educational technology in health for users of hemodynamics on coronary angiography exam: descriptive study. Journal of Nursing UFPE on line 2016;10(10):3768-3777. doi: 10.5205/reuol.9667-87805-1ED1010201607

9. Garçon L, Khasnabis C, Walker L, Nakatani Y, Lapitan J, Borg J, Berumen AV. Medical and Assistive Health Technology: Meeting the Needs of Aging Populations. The Gerontologist 2016;56(Suppl 2). doi:10.1093/geront/gnw005

10. Gehling CR, Esteves DM, Rodrigues E, Rocha ET, Silva IM, Gomes JC, Rosa MSO. Uma experiência de Educação Ambiental aplicada a trabalhadores do Sistema Único de Saúde. Disciplinarum Scientia| Saúde 2016;16(1):181-190.

11. Struchiner M, Ramos P, Serpa Junior ODD. Desenvolvimento e implementação de um ambiente virtual de aprendizagem na área da saúde: uma experiência de pesquisa baseada em design. Interface-Comunicação, Saúde, Educação 2016;20(57):485-496. doi: 10.1590/1807-57622015.0676

12. Sabóia VM, Moniz MA, Daher DV, Rangel ET, Moura JMB,
Sá FC. Dinâmica comunicativa: avaliação da tecnologia educacional sobre drogas com estudantes universitários de enfermagem [Communication dynamics: evaluation of educational technology with college nursing students]. Revista Enfermagem UERJ 2016;24(1). doi: 10.12957/ reuerj.2016.7840

13. Jorge AAF. Significados e sentidos acerca de trajetórias de desenvolvimento humano na graduação em enfermagem: contribuições para o ensino na saúde. (tese de doutorado), 2016.

14. Minayo MCS. O desafio do conhecimento: pesquisa qualitativa em saúde. Ed. 14, São Paulo: Hucitec, 2014.

15. Giehl CT, Costa AEK, Pissaia LF, Moreschi C. A equipe de enfermagem frente ao processo de implantação da Sistematização da Assistência de Enfermagem. Revista de Enfermagem e Atenção à Saúde 2016;5(2).

16. Costa AEK, Ferla NJ, Bachi R, Moreschi C, Pissaia LF. A percepção da equipe de enfermagem acerca do atendimento prestado ao idoso hospitalizado com dor. Revista Caderno Pedagógico 2016;12(3). doi: 10.22410/issn.1983-0882. v12i3a2015.968

17. Reis ZSN, Melo MDCB, Corrêa EJ, Pereira AK, Santos DB, Alves HJ. Tecnologias digitais para o ensino em saúde: relato de experiências e a convergência para o Projeto AVAS21. Revista de Saúde Digital e Tecnologias EducacionaisRESDITE 2016;1(1).

18. Souza TAC. Silva GTR. Residência Multiprofissional em Saúde: vivências e cenários da formação. São Paulo: Martinari; 2013. Ciência \& Saúde Coletiva 2016;21(3):987988. doi: 10.1590/1413-81232015213.07712015

19. Almeida AO, Silva RP. Uso da webconferência como estratégia de ensino-aprendizagem no curso técnico em Segurança do Trabalho do IFB. 2017.

20. Stelet BP, Romano VF, Carrijo APB, Junior T, Esteves J. Reflective Portfolio: philosophical contributions to a narrative praxis in medical education. Interface-Comunicação, Saúde, Educação 2017;21(60):165-176. doi: 10.1590/180757622015.0959

21. Carvalho Lira ALB, Fernandes MIDCD, Costa IA, Costa Silva RS. Estratégia de aprimoramento do ensino do exame físico em enfermagem. Enfermagem em Foco 2016;6(1/4).

22. Santos BÉFD. Avaliação discente sobre interação ensino, serviços e comunidade em equipes de saúde integradas ao Programa Mais Médicos em estado da Amazônia. 2016. Disponível em: <http://pesquisa.bvsalud.org/pmm/resource/ pt/pesqpmm-97>. Acesso em: 07 jan. 2017.

23. Cunha M, Ribeiro O, Vieira C, Pinto F, Alves $L$, Santos $R$, Andrade V. Atitudes do enfermeiro em contexto de ensino clínico: uma revisão da literatura. Millenium 2016;(38):271-282.

24. Almeida CRS, Camargo MN, Camargo LB. Educação popular e aprendizagem baseada em problemas na EAD: uma aplicação no curso de Ciências Sociais EAD/UNIMONTES. Revista Multitexto 2017;4(2):20-26.

25. Silva AR, Barros JF, Teles LF. Aprendizagem colaborativa online: uma experiência em monitoria no programa de pósgraduação em ciências da saúde. Revista de enfermagem UFPE online 2017;11(2):749-757. doi: 10.5205/reuol.1026391568-1-RV.1102201710

26. Souza LC. A TIC na Educação: uma grande aliada no aumento da aprendizagem no Brasil. Revista Eixo 2017;5(1). doi: 10.19123/eixo.v5i1.315 